\title{
Early detection and intervention using neutrophil gelatinase-associated lipocalin (NGAL) may improve renal outcome of acute contrast media induced nephropathy: A randomized controlled trial in patients undergoing intra-arterial angiography (ANTI-CIN Study)
}

Gernot Schilcher ${ }^{1}$, Werner Ribitsch¹, Ronald Otto', Rupert H Portugaller², Franz Quehenberger ${ }^{3}$, Martini Truschnig-Wilders ${ }^{4}$, Robert Zweiker ${ }^{5}$, Philipp Stiegler ${ }^{6}$, Marianne Brodmann ${ }^{7}$, Klemens Weinhandl ${ }^{1}$ and Joerg H Horina ${ }^{*}$

\begin{abstract}
Background: Patients with pre-existing impaired renal function are prone to develop acute contrast media induced nephropathy (CIN). Neutrophil gelatinase-associated lipocalin (NGAL), a new biomarker predictive for acute kidney injury (AKI), has been shown to be useful for earlier diagnosis of CIN; however, urinary NGAL values may be markedly increased in chronic renal failure at baseline. Results from those studies suggested that urinary NGAL values may not be helpful for the clinician. An intravenous volume load is a widely accepted prophylactic measure and possibly a reasonable intervention to prevent deterioration of renal function. The aim of our study is to evaluate NGAL as an early predictor of CIN and to investigate the clinical benefit of early post-procedural i.v. hydration.
\end{abstract}

Methods/Design: The study will follow a prospective, open-label, randomized controlled design. Patients requiring intra-arterial contrast media (CM) application will be included and receive standardized, weight-based, intravenous hydration before investigation. Subjects with markedly increased urinary NGAL values after CM application will be randomized into one of two study groups. Group A will receive 3-4 ml/kg BW/h 0.9\% saline intravenously for 6 hours. Group B will undergo only standard treatment consisting of unrestricted oral fluid intake. The primary outcome measure will be CIN defined by an increase greater than $25 \%$ of baseline serum creatinine. Secondary outcomes will include urinary NGAL values, cystatin C values, contrast media associated changes in cardiac parameters such as NT-pro-BNP/troponin T, changes in urinary cytology, need for renal replacement treatment, length of stay in hospital and death.

We assume that $20 \%$ of the included patients will show a definite rise in urinary NGAL. Prospective statistical power calculations indicate that the study will have $80 \%$ statistical power to detect a clinically significant decrease of CIN of 40\% in the treatment arm if 1200 patients are recruited into the study.

Discussion: A volume expansion strategy showing a benefit from earlier intervention for patients with markedly elevated urinary NGAL values, indicating a CIN, might arise from data from this study.

Trial registration: ClinicalTrials.gov NCT01292317

\footnotetext{
* Correspondence: joerg.horina@medunigraz.at

'Division of Nephrology and Hemodialysis, Department of Internal Medicine,

Medical University of Graz, Austria

Full list of author information is available at the end of the article
} 


\section{Background}

In recent decades, the growing demand for sophisticated diagnostics in all fields of modern medicine has increased the need for contrast-enhanced imaging [1]. The amount of iodinated contrast media (CM) used worldwide cannot be even roughly estimated. While contrast-enhanced procedures are fairly safe in the healthy population, patients with pre-existing impaired renal and/or cardiac function are prone to develop acute kidney injury (AKI) due to acute contrast induced nephropathy (CIN) and so have a greater risk of death $[2,3]$. This has been shown in patients undergoing coronary angiography and intervention who sometimes end up with end-stage renal disease $[4,5]$. The effect of an immediate post-procedural hemodialysis treatment to eliminate CM load is also discussed controversially [6,7].

Until recently and for this reason, physicians have increasingly used gadolinium enhanced magnetic resonance imaging to avoid renal complications, especially in such high-risk patients. For interventional settings, however, gadolinium enhanced magnetic resonance imaging has never been an appropriate alternative.

Also, with the recognition of nephrogenic systemic sclerosis as a severe and also life-threatening gadolinium side effect without treatment options, physicians now have to weigh the risk of either investigation for the individual patient $[8,9]$. A return to iodinated $\mathrm{CM}$ enhanced investigations is now seen in many clinical settings.

A variety of investigators have tried to establish risk scores for patients before investigation $[10,11]$. To date, a reliable laboratory value or test that recognizes acute renal damage before serum creatinine increases is still being sought and might be a most helpful tool to initiate proper treatment on time. Neutrophil gelatinase-associated lipocalin (NGAL), a new biomarker predictive for acute renal injury, has been shown to be capable of earlier diagnosis in patients undergoing cardiac surgery and CIN [12-16]. NGAL so might be the desired diagnostic link between acute kidney damage and the later occurrence of elevated creatinine values. Randomized controlled trials using this "timesaver" for earlier intervention have not yet been performed, but would be highly desirable [17]. Such a study might change physicians' therapeutic strategies and improve clinical outcomes.

One major drawback of this new biomarker may be the fact that only little is known about its value and accuracy in patients with underlying chronic kidney disease (CKD), and a predictive pattern or cut-off level in patients developing "acute on chronic renal failure" has not yet been defined. Some investigators found markedly increased NGAL levels in individual patients, far above the proposed cut-offs for acute renal failure. They also discovered a significant inverse relation between NGAL and renal function defined by glomerular filtration rate (GFR) $[18,19]$. Mori et al. have speculated that constantly increased, yet stable NGAL values in CKD are the consequences of sustained production by "inflamed", but vital tubular cells [20]. Even more complicating is the fact that age and several co-morbidities, such as inflammation and chronic heart disease, common in patients with chronic renal failure, may also influence NGAL levels [21,22]. A meta-analysis by Haase et al. selected 19 relevant published articles and defined 150 $\mathrm{ng} / \mathrm{ml}$ as a possible cut-off value with a proper sensitivity and specificity [23]. This analysis also confirmed a comparable accuracy of plasma and urinary NGAL, whereas urinary NGAL testing may be even better in patients with CKD [24].

So far, an intravenous volume load is the only reasonably proven and widely accepted prophylaxis for CIN after CM application [25-28]. In the majority of the relevant randomized controlled trials, isotonic saline infusion $(0.9 \%)$ was used for volume expansion, while some authors used half-isotonic saline $(0.45 \%)$ or sodium bicarbonate at higher volumes. The benefit of this type of intervention is not a pharmacologic effect of a specific type of infusion, but rather a physical effect of volume expansion. The optimal intravenous fluid regimen regarding the type, amount, route and duration of volume application remains controversial.

Hydration regimens have differed widely among relevant randomized trials and so are not comparable [29]. Further, most studies also lacked statistical power, used different types of CM and definitions of CIN or allowed for additional prophylactic measures, such as $\mathrm{N}$-acetylcysteine or sodium bicarbonate in a varying percentage of their patients [30-38].

Worldwide, there are no generally accepted guidelines for a controlled and standardized prophylactic volume application among radiologists, cardiologists, nephrologists and other involved physicians. In other words, the beneficial effect of intravenous hydration is not used to greatest advantage.

In our study, all patients requiring intra-arterial CM application will receive a weight-based, standardized intravenous hydration before investigation. This measure of controlled pre-hydration alone might be of great benefit for our patients and differs from routine clinical practice, where patients often only receive oral fluid at best. To date, intravenous hydration is not even an established clinical standard for the so-called "high-risk patient" with e.g. diabetes and/or renal failure.

A randomized trial evaluating the clinical benefit of additional post-procedural i.v. hydration might provide a 
further benefit and could be highly valuable for routine practice.

Controlled post-procedural i.v. hydration has not been used consistently for several reasons [29]:

(a) worldwide, a large percentage of CM studies are performed on an outpatient basis;

(b) there are no clear data proving the benefit of additional post-procedural i.v. hydration, versus pre-procedural hydration only;

(c) intravenous hydration, both pre- and post-procedural, is often not performed for organizational reasons.

\section{Rationale and hypothesis}

Earlier detection of CIN by the novel urinary NGAL test method enables amelioration of renal injury by the introduction of rapid and intensified post-procedural (angiography) volume expansion.

\section{Aims of our study}

1). To evaluate the true risk of CIN in high-risk patients following intra-arterial angiography using a standardized pre-procedural protocol for intravenous volume load.

2). To evaluate the benefit of an earlier diagnosis of renal damage after investigation using urinary NGAL.

3). To investigate the effect of a well-timed and intensified post-procedural volume expansion on renal function, morbidity and mortality in patients predicted to develop CIN according to NGAL testing.

4). To study NGAL in patients with mild, moderate and severe CKD (stage 2-4), a subgroup of individuals who frequently develop CIN. At present it is still unclear whether the new biomarker NGAL is comparably effective in this particular group.

5). To define and establish a reliable cut-off value or pattern of increase for NGAL in patients with chronic renal failure and to estimate its sensitivity and specificity.

6). To assess the value of a $25 \%$ increase in serum creatinine as a definition for clinically relevant CIN.

7). To compare the value of NGAL and cystatin $C$ in predicting CIN.

8). To compare the value of the MDRD, the CKDEPI- and the Cockcroft-Gault formula in this particular patient group.

At this stage, little is known about the beneficial effect of earlier diagnosis and subsequent intervention by volume expansion on AKI due to a rise in urinary NGAL. As the time course of kidney damage is usually not apparent, contrast media induced nephropathy (CIN) is a promising condition for this question. Results from previous studies are unable to guide the clinician when baseline urinary NGAL levels exceed the normal range.

To our knowledge, this is the first clinical study to address this problem. We are trying to assess the effect of an early intervention by volume expansion in patients with increasing urinary NGAL levels 4-6 hours following $\mathrm{CM}$ application. If a significant reduction of CIN as defined by creatinine levels is found in the treatment arm within 2 days, a clear benefit of measuring urinary NGAL might be postulated. We are currently recruiting patients for this non-commercial investigator initiated trial to elucidate CIN.

\section{Methods and design}

Study Design and Setting

This study is a single center, open label, randomized controlled trial involving subjects undergoing an intraarterial angiographic procedure with iodinated $\mathrm{CM}$. The study is being conducted at the University Hospital in Graz, Austria.

\section{Ethical considerations}

The Ethics Committee of the Medical University of Graz, Austria, approved this trial (Registration Number: 21-278 ex 09/10). The Ethics Committees will be provided with annual reports of trial progress and will promptly receive all adverse event reports. Participants may declare their withdrawal at any time during the study.

\section{Participants}

Patients scheduled for diagnostic intra-arterial catheter angiography or endovascular intervention will be invited to participate in the study. Only one type of iso-osmolar, non-ionic, monomeric CM (Iomeron ${ }^{\mathbb{B}}$ ) will be used. The most common underlying diseases in these individuals are coronary heart disease, peripheral arterial occlusive disease and renal artery stenosis.

\section{Identification of eligible patients}

The principle investigator and the clinical trial coordinators will screen the medical records of patients for preexisting renal failure one day prior to an angiographic intervention at the Divisions of Nephrology, Cardiology, Angiology and Radiology at the University Hospital in Graz, Austria. Eligible patients will have a copy of the patient information sheet placed in the medical record. The investigator will explain the study during the clinical consultation. Only subjects giving written informed consent will be included. The study period will last 4 days, starting one day prior to investigation and ending two days after the intra-arterial angiography (Figure 1). A one year follow up consultation is targeted. All patients with CIN will be followed separately. 


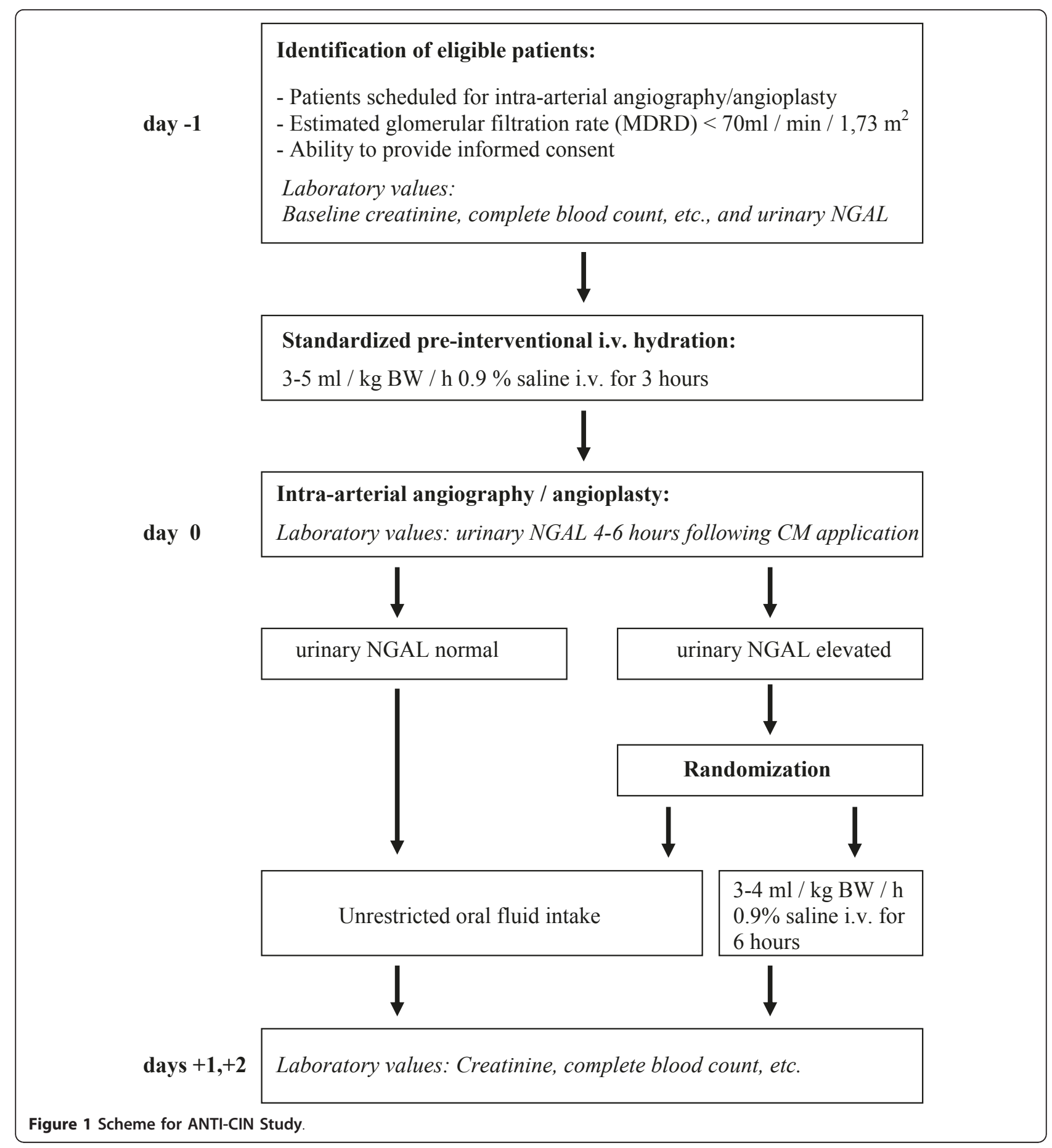

Inclusion criteria

- Patients requiring intra-arterial angiography/ angioplasty

- Patients older than 18 years

- Patients with clinically stable CKD of stage 2 or more (calculated GFR $<70 \mathrm{ml} / \mathrm{min} / 1,73 \mathrm{~m} 2)(65)$

- Gender: both
- Written informed consent

\section{Exclusion criteria}

- Pre-existing clinical and/or laboratory evidence of acute renal failure at the time of enrolment

- Evidence of rhabdomyolysis 
- Patients on renal replacement therapy (34)

- Patients with life-threatening underlying disease

- Contraindication for volume therapy

- Pregnancy

- $\mathrm{CM}$ application within 7 days prior to intervention

\section{Standardized procedure}

Hospitalized patients scheduled for elective intra-arterial angiography will be prepared for $\mathrm{CM}$ application using a weight-based hydration protocol.

Monomeric Iomeron ${ }^{\circledR}$ (iomeprol, Bracco Austria, Vienna) will be used exclusively as contrast media. Blood samples for standard evaluation, including serum creatinine and cystatin $\mathrm{C}$, will be drawn at baseline and after 24 and 48 hours from all patients. A BW/h saline infusion (3-5 ml/ kg) will be given for 3 hours before $C M$ application. Urine samples for NGAL testing will be collected shortly before (baseline) and after 4 to 6 hours following CM application. NGAL will be measured with the commercially available ARCHITECT ${ }^{\circledR}$ NGAL Test, Abbott Laboratories, Abbott Park, Illinois, U.S.A. [15,39].

Patients who develop CIN will be treated and followed at the Division of Nephrology and Hemodialysis according to established standards.

\section{Randomization}

Patients will be allocated randomly using a Web-based computer program http://www.randomizer.at. Balance of treatment counts is achieved by the biased-coin method with probability 0.7 [40]. Randomization will occur shortly after receipt of the second urinary NGAL value. NGAL determinations will be made by the medical technical staff of the Clinical Institute for Laboratory Medicine, Medical University of Graz, Austria. The criteria for randomization (after $2^{\text {nd }}$ NGAL testing, $4-6$ hours following $\mathrm{CM}$ application) are:

- Patients with NGAL levels > $150 \mathrm{ng} / \mathrm{ml}$, if baseline was below $75 \mathrm{ng} / \mathrm{ml}$ [23].

- Patients with doubling of NGAL values, if baseline values were $>75 \mathrm{ng} / \mathrm{ml}[18]$.

\section{Procedure following randomization}

Study patients with a defined increase of urinary NGAL values at 4 to 6 hours after CM application will be randomized into one of two groups:

Patients of Group A will receive 3-4 ml/ $\mathrm{kg} \mathrm{BW/h}$ $0.9 \%$ saline intravenously for 6 hours post procedure in addition to oral fluid.

Patients of Group B will receive unrestricted oral fluid. An intake of at least $500 \mathrm{ml}$ tea or water is advised and provided on the ward.

\section{Blinding}

Blinding of investigators and patients is not feasible as it is immediately recognizable and impossible to conceal that only one group (Group A) receives saline intravenously.

\section{Outcome measures}

\section{Primary outcome measure}

CIN defined by an increase greater than $25 \%$ of baseline serum creatinine. Additionally, patients will be classified according to the Cockcroft and Gault, as well as the MDRD and the CKD-EPI calculation formula.

\section{Secondary outcome measures}

Urinary NGAL values, cystatin $C$ values, contrast media associated changes of cardiac parameters such as NTpro-BNP/Troponin $\mathrm{T}$, changes in urinary cytology, need for renal replacement treatment, length of hospital stay and death.

\section{Monitoring for adverse events}

The study investigators and the Coordination Center for Clinical Studies, Medical University of Graz, authored a standardized operating procedure for reporting adverse events. Although this trial has not been declared as a pharmacological interventional study, because the volume expansion is considered a physical rather than a pharmacological effect, the investigators voluntarily chose to report adverse events according to the guidelines for a pharmacological interventional study. All relevant adverse events will be summarized and reported to the Ethics Committee of the Medical University of Graz.

\section{Data collection}

Historical data will be obtained from the patient or collected from medical records. These include age, gender, weight, height, relevant co-morbidities, existence of peripheral edema, current medication, contrast media dose and location of angiography/angioplasty/stent implantation. Furthermore, there will be repeated lab studies including complete blood count, electrolytes, serum creatinine, coagulation profile, liver function test, Creactive protein, bicarbonate and spot urine analysis and urinary NGAL. Length of hospitalization will documented. All data are stored in an electronic case report form.

\section{Sample size calculations}

Sample Size: Alpha $=0.05$, power $=0.8$, two-sided test. Assuming a clinically relevant difference in CIN incidence of $20 \%$ and $60 \%$ CIN in the control group (worst case), 108 patients per group are required for the chi square test. Only patients who meet the NGAL criteria for randomization will be included. We assume that $20 \%$ of the included patients, which would amount to 
about 1200 patients, will be randomized to treatment groups. If fewer than 48 patients are randomized after the inclusion of 240 patients, the NGAL criteria for randomization will be adapted in order to meet the objective of including not more than 1200 patients. Recruitment will continue until 240 patients have been randomized.

Analysis: Chi square tests will be used for CIN reduction. Wilcoxon's rank sum test will be applied to continuous measurements.

\section{Statistical considerations}

The diagnostic value of a two-point-measurement of NGAL, cystatin $C$ and creatinine before and after CM application, as compared to a single-point-measurement only after CM application with respect to CIN will be assessed through receiver operating characteristic (ROC) within the patients who received standard therapy. The analysis has to account for the bias that is caused by the exclusion of patients who receive treatment A from ROC analysis on the condition of an elevated NGAL. The available sample size for ROC analysis is determined by the number of patients, the incidence of CIN and the number of patients who receive treatment $\mathrm{A}$. We assume that $20 \%$ of the included patients will develop CIN.

\section{Data analysis}

Treatments will be compared according to relative change of creatinine from baseline by the Wilcoxon test and Pearson's chi square test (two-sided, alpha $=0.05$ ).

As mentioned above, those patients without a relevant increase of NGAL will also be followed. This will allow us to retrospectively calculate sensitivity and specificity of NGAL testing for CIN.

\section{Discussion}

To the best of our knowledge there is no other interventional study published or ongoing using "timesaving" effect due to NGAL evaluation to prevent CIN http:// www.clinicaltrials.gov.

Though a controlled study evaluating the benefit of early diagnosis and intervention would be highly desirable, the planning of such a study has some major difficulties and drawbacks:

1. An established cut-off for NGAL has not been defined for patients with CKD. Many investigators have found a wide range from approximately 5 to $800 \mathrm{ng} / \mathrm{ml}$ in such patients. We therefore believe that at present, one single-point measurement may be insufficient to differentiate between acute and chronic disease and so will perform 2-point measurements. It is hoped that inter-individual changes before and after investigation will be of great help in this differentiation.
2. As an increase of NGAL after cardiopulmonary bypass in children (without pre-existing renal disease or nephrosclerosis) has been found without development of AKI, a mild increase of NGAL values will not lead to randomization [15].

3. Since increased baseline values above the established cut-off for AKI should not lead to exclusion from the study of patients with CKD, we have developed subtle criteria for randomization. It is our hypothesis that at least doubling of NGAL should be demanded in patients with CKD, whereas in patients with very low values at baseline even a higher-fold increase might be without clinical relevance, as long as values stay below $150 \mathrm{ng} / \mathrm{ml}$.

4. To define study size and patient numbers it would be necessary to know the percentage of patients who will show NGAL increase. In such a high-risk patient group, we would estimate that more than 15 to 20 percent of patients might develop CIN, based on the contrast media literature. A fine-tuning of cut-off levels after intermediate analysis would be statistically possible without patient exclusions.

5. The effect of intensified hydration is not clearly established, although data support its benefit.

6. Urinary NGAL seems to be even better than plasma NGAL. The Architect kit showed reliable results [15].

In summary, a clearly defined and simplified prophylactic volume expansion strategy showing a benefit of earlier intervention in patients with markedly elevated urinary NGAL values, indicating CIN, might arise from the data this study produces.

\section{List of abbreviations}

CM: contrast media; AKI: acute kidney injury; CIN: contrast media induced nephropathy; NGAL: neutrophil gelatinase-associated Lipocalin; CKD: chronic kidney disease

\section{Acknowledgements and funding}

We are grateful to Abbott GmbH \& Co. KG (Germany) for providing essential laboratory test material (urinary neutrophil gelatinase-associated LipocalinNGAL) as well as financial funding for two study nurses over the study period. The participation of patients and the assistance of the nursing staff at the University Hospital in Graz, Austria are greatly appreciated.

\section{Author details}

'Division of Nephrology and Hemodialysis, Department of Internal Medicine, Medical University of Graz, Austria. ${ }^{2}$ Department of Radiology, Medical University of Graz, Austria. ${ }^{3}$ Institute for Medical Informatics, Statistics and Documentation, Medical University of Graz, Austria. ${ }^{4}$ Clinical Institute for Laboratory Medicine, Medical University of Graz, Austria. ${ }^{5}$ Division of Cardiology, Department of Internal Medicine, Medical University of Graz, Austria. ${ }^{6}$ Division of Transplantation Surgery, Department of Surgery, Medical University of Graz, Austria. ${ }^{7}$ Division of Angiology, Department of Internal Medicine, Medical University of Graz, Austria.

\section{Authors' contributions}

$\mathrm{JHH}$ is the principle investigator of this non-commercial investigator initiated study. JHH, GS, WR, RO and KW are responsible for the design of this clinical trial, the construction of the protocol and writing of the manuscript. FQ provided the sample size calculation and the randomization process and 
critically reviewed the study design and the protocol in terms of statistical aspects. He will also perform the data analysis. HP, MTW, RZ, PS and MB took part in initiating the study, helped to draft the manuscript as experts in their fields and are currently part of the trial managing team. All authors read and approved the final manuscript.

\section{Competing interests}

The authors declare that they have no competing interests.

Received: 5 March 2011 Accepted: 17 August 2011

Published: 17 August 2011

\section{References}

1. Toms AP, Cash CJ, Linton SJ, Dixon AK: Requests for body computed tomography: increasing workload, increasing indications and increasing age. Eur Radiol 2001, 11(12):2633-2637.

2. Tepel M, Aspelin P, Lameire N: Contrast-induced nephropathy: a clinical and evidence-based approach. Circulation 2006, 113(14):1799-1806.

3. Solomon R, Barrett B: Follow-up of patients with contrast-induced nephropathy. Kidney Int Supp/ 2006, 100(100):S46-50.

4. McCullough PA, Wolyn R, Rocher LL, Levin RN, O'Neill WW: Acute renal failure after coronary intervention: incidence, risk factors, and relationship to mortality. Am J Med 1997, 103(5):368-375.

5. Rihal CS, Textor SC, Grill DE, Berger PB, Ting HH, Best PJ, Singh M, Bell MR, Barsness GW, Mathew V, Garratt KN, Holmes DR Jr: Incidence and prognostic importance of acute renal failure after percutaneous coronary intervention. Circulation 2002, 105(19):2259-2264.

6. Vogt B, Ferrari P, Schonholzer C, Marti HP, Mohaupt M, Wiederkehr M, Cereghetti C, Serra A, Huynh-Do U, Uehlinger D, Frey FJ: Prophylactic hemodialysis after radiocontrast media in patients with renal insufficiency is potentially harmful. Am J Med 2001, 111(9):692-698.

7. Marenzi G, Marana I, Lauri G, Assanelli E, Grazi M, Campodonico J, Trabattoni D, Fabbiocchi F, Montorsi P, Bartorelli AL: The prevention of radiocontrast-agent-induced nephropathy by hemofiltration. $N$ Engl J Med 2003, 349(14):1333-1340.

8. Kane GC, Stanson AW, Kalnicka D, Rosenthal DW, Lee CU, Textor SC, Garovic VD: Comparison between gadolinium and iodine contrast for percutaneous intervention in atherosclerotic renal artery stenosis: clinical outcomes. Nephrol Dial Transplant 2008, 23(4):1233-1240.

9. Grobner T: Gadolinium-a specific trigger for the development of nephrogenic fibrosing dermopathy and nephrogenic systemic fibrosis? Nephrol Dial Transplant 2006, 21(4):1104-1108.

10. Fortescue EB, Bates DW, Chertow GM: Predicting acute renal failure after coronary bypass surgery: cross-validation of two risk-stratification algorithms. Kidney Int 2000, 57(6):2594-2602.

11. Thakar CV, Arrigain S, Worley S, Yared JP, Paganini EP: A clinical score to predict acute renal failure after cardiac surgery. J Am Soc Nephrol 2005, 16(1):162-168.

12. Hirsch $R$, Dent $C$, Pfriem H, Allen J, Beekman $R H$, Ma Q, Dastrala S, Bennett $M$, Mitsnefes M, Devarajan P: NGAL is an early predictive biomarker of contrast-induced nephropathy in children. Pediatr Nephrol 2007, 22(12):2089-2095.

13. Mishra J, Dent C, Tarabishi R, Mitsnefes MM, Ma Q, Kelly C, Ruff SM, Zahedi K, Shao M, Bean J, Mori K, Barasch J, Devarajan P: Neutrophil gelatinase-associated lipocalin (NGAL) as a biomarker for acute renal injury after cardiac surgery. Lancet 2005, 365(9466):1231-1238.

14. Nickolas TL, O'Rourke MJ, Yang J, Sise ME, Canetta PA, Barasch N, Buchen C, Khan F, Mori K, Giglio J, Devarajan P, Barasch J: Sensitivity and specificity of a single emergency department measurement of urinary neutrophil gelatinase-associated lipocalin for diagnosing acute kidney injury. Ann Intern Med 2008, 148(11):810-819.

15. Bennett M, Dent CL, Ma Q, Dastrala S, Grenier F, Workman R, Syed H, Ali S, Barasch J, Devarajan P: Urine NGAL predicts severity of acute kidney injury after cardiac surgery: a prospective study. Clin J Am Soc Nephrol 2008, 3(3):665-673.

16. Haase-Fielitz A, Bellomo R, Devarajan P, Story D, Matalanis G, Dragun D, Haase M: Novel and conventional serum biomarkers predicting acute kidney injury in adult cardiac surgery-a prospective cohort study. Crit Care Med 2009, 37(2):553-560.
17. Parikh CR, Garg AX: Testing new biomarkers for acute kidney injury: association, prediction, and intervention. Am J Kidney Dis 2009, 54(6):987-989

18. Bolignano D, Lacquaniti A, Coppolino G, Donato V, Campo S, Fazio MR, Nicocia G, Buemi M: Neutrophil gelatinase-associated lipocalin (NGAL) and progression of chronic kidney disease. Clin J Am Soc Nephrol 2009, 4(2):337-344.

19. Bolignano D, Lacquaniti A, Coppolino G, Campo S, Arena A, Buemi M: Neutrophil gelatinase-associated lipocalin reflects the severity of renal impairment in subjects affected by chronic kidney disease. Kidney Blood Press Res 2008, 31(4):255-258.

20. Mori K, Nakao K: Neutrophil gelatinase-associated lipocalin as the realtime indicator of active kidney damage. Kidney Int 2007, 71(10):967-970.

21. Malyszko J, Malyszko JS, Koc-Zorawska E, Kozminski P, Mysliwiec M: Neutrophil gelatinase-associated lipocalin in dialyzed patients is related to residual renal function, type of renal replacement therapy and inflammation. Kidney Blood Press Res 2009, 32(6):464-469.

22. Poniatowski B, Malyszko J, Bachorzewska-Gajewska H, Malyszko JS, Dobrzycki S: Serum neutrophil gelatinase-associated lipocalin as a marker of renal function in patients with chronic heart failure and coronary artery disease. Kidney Blood Press Res 2009, 32(2):77-80

23. Haase M, Bellomo R, Devarajan P, Schlattmann P, Haase-Fielitz A, NGAL Meta-analysis Investigator Group: Accuracy of neutrophil gelatinaseassociated lipocalin (NGAL) in diagnosis and prognosis in acute kidney injury: a systematic review and meta-analysis. Am J Kidney Dis 2009, 54(6):1012-1024.

24. Nishida M, Kawakatsu H, Okumura Y, Hamaoka K: Serum and urinary neutrophil gelatinase-associated lipocalin levels in children with chronic renal diseases. Pediatr Int 2010, 52(4):563-568.

25. Weisbord SD, Palevsky PM: Prevention of contrast-induced nephropathy with volume expansion. Clin J Am Soc Nephrol 2008, 3(1):273-280.

26. Mueller C, Buerkle G, Buettner HJ, Petersen J, Perruchoud AP, Eriksson U, Marsch S, Roskamm H: Prevention of contrast media-associated nephropathy: randomized comparison of 2 hydration regimens in 1620 patients undergoing coronary angioplasty. Arch Intern Med 2002, 162(3):329-336.

27. Persson PB, Patzak A: Renal haemodynamic alterations in contrast medium-induced nephropathy and the benefit of hydration. Nephrol Dial Transplant 2005, 20(Suppl 1):i2-5.

28. Bader BD, Berger ED, Heede MB, Silberbaur I, Duda S, Risler T, Erley CM: What is the best hydration regimen to prevent contrast media-induced nephrotoxicity? Clin Nephrol 2004, 62(1):1-7

29. Reddan D, Laville M, Garovic VD: Contrast-induced nephropathy and its prevention: What do we really know from evidence-based findings? J Nephrol 2009, 22(3):333-351.

30. Solomon R, Werner C, Mann D, D'Elia J, Silva P: Effects of saline, mannitol, and furosemide to prevent acute decreases in renal function induced by radiocontrast agents. N Engl J Med 1994, 331(21):1416-1420.

31. Merten GJ, Burgess WP, Gray LV, Holleman JH, Roush TS, Kowalchuk GJ, Bersin RM, Van Moore A, Simonton CA, Rittase RA, Norton HJ, Kennedy TP. Prevention of contrast-induced nephropathy with sodium bicarbonate: a randomized controlled trial. JAMA 2004, 291(19):2328-2334.

32. Briguori C, Airoldi F, D'Andrea D, Bonizzoni E, Morici N, Focaccio A, Michev I, Montorfano M, Carlino M, Cosgrave J, Ricciardelli B, Colombo A: Renal Insufficiency Following Contrast Media Administration Trial (REMEDIAL): a randomized comparison of 3 preventive strategies. Circulation 2007, 115(10):1211-1217.

33. Recio-Mayoral A, Chaparro M, Prado B, Cozar R, Mendez I, Banerjee D, Kaski JC, Cubero J, Cruz JM: The reno-protective effect of hydration with sodium bicarbonate plus $\mathrm{N}$-acetylcysteine in patients undergoing emergency percutaneous coronary intervention: the RENO Study. J Am Coll Cardiol 2007, 49(12):1283-1288.

34. Tepel M, van der Giet M, Schwarzfeld C, Laufer U, Liermann D, Zidek W: Prevention of radiographic-contrast-agent-induced reductions in renal function by acetylcysteine. N Engl J Med 2000, 343(3):180-184.

35. Marenzi G, Assanelli E, Marana I, Lauri G, Campodonico J, Grazi M, De Metrio M, Galli S, Fabbiocchi F, Montorsi P, Veglia F, Bartorelli AL: Nacetylcysteine and contrast-induced nephropathy in primary angioplasty. N Engl J Med 2006, 354(26):2773-2782. 
36. Aspelin P, Aubry P, Fransson SG, Strasser R, Willenbrock R, Berg K,

Nephrotoxicity in High-Risk Patients Study of Iso-Osmolar and Low-Osmolar Non-Ionic Contrast Media Study Investigators: Nephrotoxic effects in highrisk patients undergoing angiography. N Engl J Med 2003, 348(6):491-499.

37. Jo SH, Youn TJ, Koo BK, Park JS, Kang HJ, Cho YS, Chung WY, Joo GW, Chae IH, Choi DJ, Oh BH, Lee MM, Park YB, Kim HS: Renal toxicity evaluation and comparison between visipaque (iodixanol) and hexabrix (ioxaglate) in patients with renal insufficiency undergoing coronary angiography: the RECOVER study: a randomized controlled trial. J Am Coll Cardiol 2006, 48(5):924-930.

38. Briguori C, Colombo A, Violante A, Balestrieri P, Manganelli F, Paolo Elia P, Golia B, Lepore S, Riviezzo G, Scarpato P, Focaccio A, Librera M, Bonizzoni E, Ricciardelli B: Standard vs double dose of $\mathrm{N}$-acetylcysteine to prevent contrast agent associated nephrotoxicity. Eur Heart J 2004, 25(3):206-211.

39. Grenier FC, Ali S, Syed H, Workman R, Martens F, Liao M, Wang Y, Wong PY: Evaluation of the ARCHITECT urine NGAL assay: assay performance, specimen handling requirements and biological variability. Clin Biochem 2010, 43(6):615-620.

40. Efron B: Forcing a sequential experiment to be balanced. Biometrika 1971, 58(3):403-417.

\section{Pre-publication history}

The pre-publication history for this paper can be accessed here: http://www.biomedcentral.com/1471-2369/12/39/prepub

\section{doi:10.1186/1471-2369-12-39}

Cite this article as: Schilcher et al: Early detection and intervention using neutrophil gelatinase-associated lipocalin (NGAL) may improve renal outcome of acute contrast media induced nephropathy: A randomized controlled trial in patients undergoing intra-arterial angiography (ANTI-CIN Study). BMC Nephrology 2011 12:39.

\section{Submit your next manuscript to BioMed Central and take full advantage of:}

- Convenient online submission

- Thorough peer review

- No space constraints or color figure charges

- Immediate publication on acceptance

- Inclusion in PubMed, CAS, Scopus and Google Scholar

- Research which is freely available for redistribution

Submit your manuscript at www.biomedcentral.com/submit 